\title{
Prognose der Leistungsverfügbarkeit während der Planung
}

\author{
Dipl.-Ing. Martina M. Maier \\ Technische Universität IImenau \\ Institut für rechnerunterstützte Produktion
}

\begin{abstract}
Der Fachausschuss Zuverlässigkeit in der Intralogistik der VDIGesellschaft Produktion und Logistik (VDI-GPL) veröffentlicht Ende 2010 die VDIRichtlinie 4486: „Zuverlässigkeit in der Intralogistik - Leistungsverfügbarkeit“ [VDI04].

Der Kennwert der Leistungsverfügbarkeit wurde insbesondere für die Anforderungen des Verfügbarkeitstests im Rahmen der Abnahme entwickelt und stellt eine Alternative zu konventionellen Verfahren dar, bei dem aus den Verfügbarkeiten der Anlagenelemente die Gesamtverfügbarkeit berechnet wird [VDI04, VDI92]. Möglichkeiten der konkreten Ausgestaltung der Verfügbarkeitstests wurden im Rahmen des 5. WGTLKolloquiums vorgestellt (vgl. [Mai09]). Im folgenden Beitrag wird eine Methode entwickelt, mit der der Kennwert bereits in der Planungsphase abgeschätzt werden kann.
\end{abstract}

Anhand von drei Auslegungsvarianten eines Hochregallagers mit Vorzone inklusive Erweiterungsstufe wird die Anwendung der Methode beispielhaft aufgezeigt.

Schlagworte: Leistungsverfügbarkeit, Planung von intralogistischen Anlagen, Prognosemethode, VDI 4486

\section{Der Kennwert „Leistungsverfügbarkeit“}

Die Leistungsverfügbarkeit gibt den anforderungs- und termingerechten Erfüllungsgrad von zwischen Vertragspartnern (Hersteller und Anwender) vereinbarten Prozessen unter Einhaltung der vereinbarten Rahmenbedingungen an [VDI10].

Zur Quantifizierung des Erfüllungsgrades werden in der Richtlinie zwei Varianten definiert: Wird der Schaden durch die Nichtverfügbarkeit eines Prozesses stetig mit seiner Dauer größer, wird die Leistungsverfügbarkeit mit der Wartezeit an den entsprechenden Schnittstellen gemessen. Die Leistungsverfügbarkeit $\eta_{W}$ an einem Arbeitsplatz berechnet sich in diesem Fall aus der Betriebszeit $T_{B}$ und der Wartezeit $T_{W}$ wie folgt:

$$
\eta_{W}=\frac{T_{B}-T_{W}}{T_{B}}
$$

Tritt ein Schaden ein, wenn der Prozess zu einem bestimmten Zeitpunkt nicht abgeschlossen wurde und verändert sich dann nicht mehr mit fortschreitender Zeit, berechnet sich die Leistungsverfügbarkeit $\eta_{L}$ über die Stückzahl $n$ der Güter, die nicht rechtzeitig zu ihrem Ziel gefördert wurden ( $N$ : Gesamtstückzahl):

$$
\eta_{L}=\frac{N-n}{N}
$$


Im Unterschied zu den konventionellen Methoden, haben die Verfügbarkeitswerte der Elemente einer intralogistische Anlage und deren Verknüpfung keinen Einfluss auf die Messung der Leistungsverfügbarkeit. Gleichwohl spielt die Wahl eines bedarfsgerechten Layouts mit sinnvollem Einsatz von Redundanzen und Reservekapazitäten eine wichtige Rolle bei der erreichbaren Leistungsverfügbarkeit. Um verschiedene technische Varianten im Hinblick auf die zu erwartende Leistungsverfügbarkeit vergleichen zu können, wird im Folgenden eine Prognosemethode vorgestellt.

\section{Die Prognosemethode}

Das Konzept der Leistungsverfügbarkeit wurde für komplexe intralogistische Anlagen mit folgenden Voraussetzungen entwickelt:

- definierbare(r) Geschäftsprozess(e)

- messbare Kenngrößen der Geschäftsprozesse

- Berücksichtigung von Puffern, Redundanzen und Leistungsreserven

- Verantwortung für die Gesamtanlage liegt in einer Hand (Generalunternehmer)

Für die Anwendung der Prognosemethode müssen weitere Einschränkungen getroffen werden: Die Transportwege und Durchlaufzeiten der Gütermüssen relativ ähnlich sein. Gepäckförderanlagen beispielsweise erfüllen dieses Kriterium nicht, da die Gepäckstücke von vielen verschieden Quellen (Schalter, Transfergepäck) über unterschiedliche Transportstrecken (evtl. Frühgepäckspeicher, verschiedene Durchlaufzeiten) zu unterschiedlichen Zeitpunkten (abhängig vom Abflugzeitpunkt) an vielen verschiedenen Senken (Gates) transportiert werden müssen.

Für die Prognose der Wartezeit bzw. der unpünktlichen Güter werden vier Parameter berücksichtigt: Der Redundanzfaktor $R_{i}$, der Kapazitätsfaktor $K_{i x}$, die Pufferzeit $P_{i x}$ und die Laufzeitreserve $L_{i x}$.

\subsection{Redundanzfaktor}

Der Redundanzfaktor $R_{i}$ berücksichtigt den Materialflussanteil, der an das betrachtete Element $i$ gebunden ist und auch im Störungsfall nicht über redundante Strecken umgeleitet werden kann. Wird also beispielsweise ein Materialfluss über zwei parallele, gleichartige Strecken geleitet, die jeweils $75 \%$ des gesamten Materialflusses aufnehmen können, ist im Störungsfall einer der Parallelstrecken nur 25\% des Materialflusses blockiert. Der Redundanzfaktor der beiden Strecken ist demnach 0,25. Der Redundanzfaktor vermindert einerseits den Anstieg von Wartezeiteinheit bzw. Anzahl unpünktlicher Güter pro Störungszeiteinheit und vergrößert andererseits die Pufferwirkung (siehe Abschnitt 2.3).

Wirkt sich allerdings eine Störung gleichzeitig auf $m$ gleichartige und gemeinsam betrachtete Schnittstellen aus, so nimmt die Störungsauswirkung an den Schnittstellen in Summe auch $m$-fach zu. 


\subsection{Kapazitätsfaktor}

Um Spitzenlasten abfangen zu können, sind intralogistische Anlagen üblicherweise mit Reservekapazitäten ausgestattet, Die Anlagenelemente sind damit in der Lage, eine höhere Leistung (Spielzeiten, Durchsatz) zu bringen, als im Durchschnitt verlangt. Diese Reservekapazitäten können einerseits nach behobener Störung die entstandene Warteschlange mit erhöhter Leistung abarbeiten und damit die Auswirkung der Störungszeit auf die unpünktlichen Güter vermindern, und andererseits bei parallelen Anlagenelementen im Störungsfall Materialflussanteile übernehmen, wenn dies von der Anlagensteuerung so vorgesehen ist. Entscheidend für die schnellere Abarbeitung der Warteschlange ist allerdings nicht der Kapazitätsfaktor des betrachteten Elements, sondern der kleinste Kapazitätsfaktor zwischen dem betrachteten Element $i$ und der betrachteten Schnittstelle $x$, da der gesteigerte Durchsatz durch diese Engstelle begrenzt ist. Tabelle 1 zeigt die Reservekapazitäten von drei Elementen vor einer Schnittstelle $x$ und die jeweils wirksamen Reservekapazitäten.

Tabelle 1: wirksame Reservekapazitäten

\begin{tabular}{c|ccc} 
Element & $E_{1}$ & $E_{2}$ & $E_{3}$ \\
\hline Reservekapazitätsfaktor Ki & 2 & 1,5 & 2,5 \\
auf $\mathrm{x}$ wirksamer Reservekapazitätsfaktor Kix & 1,5 & 1,5 & 2,5
\end{tabular}

\subsection{Pufferzeit}

Puffer können die Auswirkungen von Störungen mindern, da während der Störung vom gepufferten Arbeitsvorrat oder in den Entsorgungspuffer gearbeitet werden kann. Ist die Störung kürzer als die Pufferzeit, so tritt an der Schnittstelle gar keine Wartezeit auf, ist die Störung länger als die Pufferzeit, wird nur die Differenz aus Störungszeit und Pufferzeit zur Wartezeit. Die Pufferzeit berechnet sich aus der Anzahl der Pufferplätze $p_{i x}$ zwischen gestörtem Element und betrachteter Schnittstelle und dem Arbeitstakt $t_{x}$ an der Schnittstelle. Bei Redundanzen verstärkt sich die Pufferwirkung um den Materialflussanteil, der den Puffer weiter ver- bzw. entsorgt.

$$
P_{i x}=t_{x} \cdot p_{i x} \cdot\left(2-R_{i}\right)
$$

\subsection{Laufzeitreserve}

Wird die Leistungsverfügbarkeit über das Kriterium „Anzahl nicht termingerechter Einheiten" gemessen, ist jede Einheit mit einem stetig sinkenden Zeitvorrat bis zum Ende des Betrachtungszeitraums ausgestattet. Gerät eine Einheit in einen Stau in Folge einer Störung, so minimiert sich der Zeitvorrat durch die Verzögerung im Stau. Ist die Verzögerung größer als der Zeitvorrat, wird die Einheit nicht termingerecht an der Zielschnittstelle eintreffen. Die Laufzeitreserve $L_{i x}$ beschreibt die Zeitdauer, die ein Ausfall maximal annehmen darf, sodass gerade noch alle Fördereinheiten 
termingerecht ihre Schnittstelle erreichen. Sie ist abhängig von der Reservezeit $T_{R}$ von Startzeitpunkt der Störung bis Ende der Betrachtungsdauer $T_{B}$ und dem Reservekapazitätsfaktor $K_{i x}$.

Die Laufzeitreserve berechnet sich nach

$$
L_{i x}=\frac{T_{R}\left(K_{i x}-1\right)}{K_{i x}}
$$

Da bei Prognose der Störungszeitpunkt nicht bekannt ist, wird der Zeitpunkt in der Mitte, also $T_{R}=0,5 \cdot T_{B}$ empfohlen, da die Hälfte aller Störungen vor diesem Zeitpunkt und die andere Hälfte nach diesem Zeitpunkt zu erwarten sind und sich so automatisch ein Mittelwert ergibt.

\subsection{Prognosegleichungen}

Zusammengefasst ergeben sich aus den Einflussgrößen die Gleichung (5) zur Wartezeitprognose und Gleichung (6) zur Prognose der unpünktlichen Güter:

$$
\begin{aligned}
& T_{W, i x}= \begin{cases}0 & f \ddot{\mathrm{u}} r T_{A i}<P_{i x} \\
\frac{R_{i}}{K_{i x}}\left(T_{A i}-P_{i x}\right) & \text { für } T_{A i} \geq P_{i x}\end{cases} \\
& n_{i x}= \begin{cases}0 & \text { für } T_{A i}<P_{i x}+L_{i x} \\
\frac{R_{i}}{K_{i x} \cdot t}\left(T_{A i}-P_{i x}-L_{i x}\right) & \text { für } T_{A i} \geq P_{i x}+L_{i x}\end{cases}
\end{aligned}
$$

Da die Anzahl der unpünktlichen Güter nur eine Ganzzahl sein kann, wird jeweils abgerundet. Die Graphen der beiden Funktionsgleichungen lassen sich demnach charakterisieren durch einen Totzeitanteil und eine linearen Anstieg der Wartezeit bzw. der Anzahl der unpünktlichen Güter mit wachsender Störungszeit (vgl. Abbildung 1 und 2).

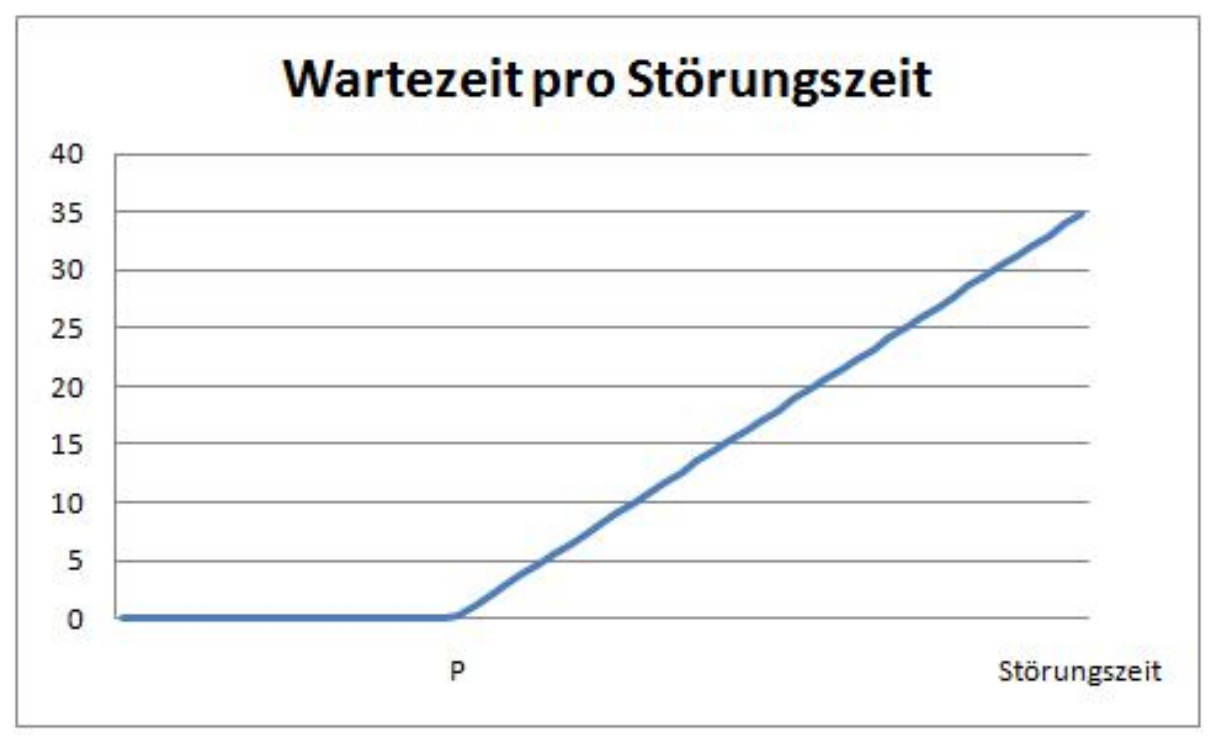

Abbildung 1: Entwicklung der Wartezeit 


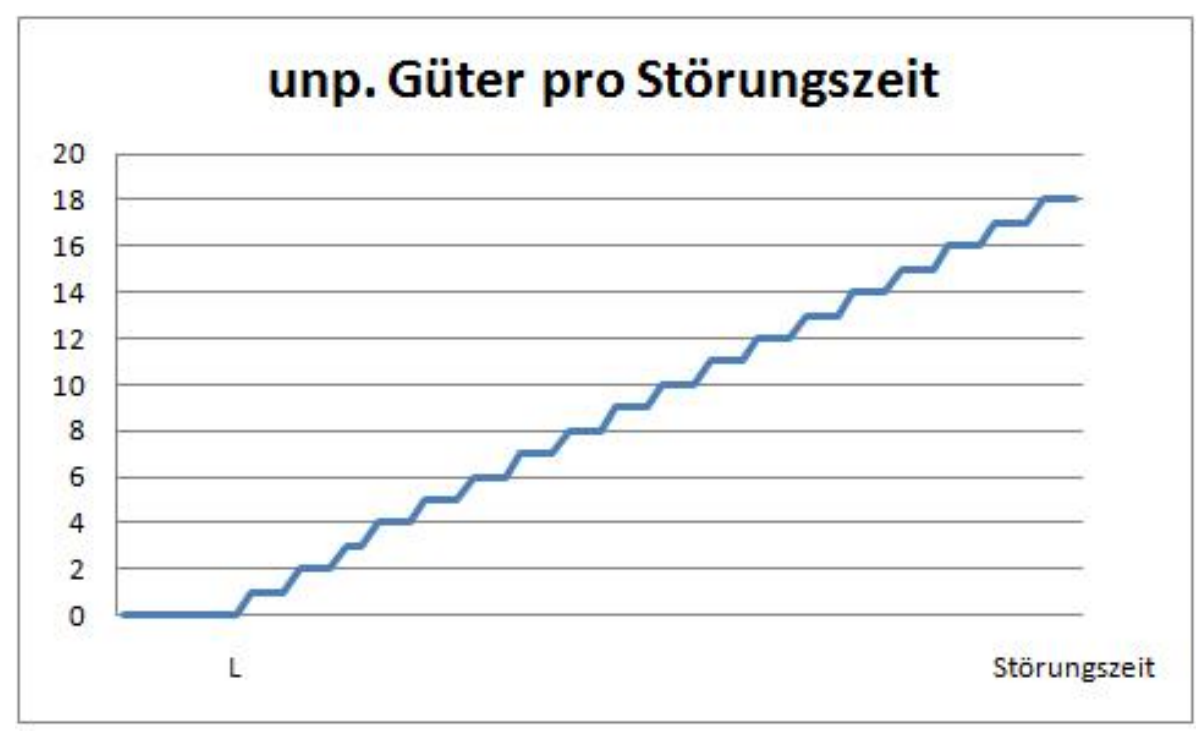

Abbildung 2: Entwicklung der pünktlichen Güter

\subsection{Auswertung}

Setzt man die maximal tolerierten Wartezeiten, bzw. unpünktlichen Güter aus den Vereinbarungen in die Gleichungen (5) und (6) kann die jeweils kritische Ausfallzeit der Anlagenelemente berechnet werden.

Diese Werte können dann beispielsweise in den Vergleich verschiedener technischer Varianten mit einbezogen werden oder zur Auswahl geeigneter Zukaufkomponenten genutzt werden.

\section{$3 \quad$ Validierung der Methode}

Im Rahmen einer Bachelorarbeit [Nag10] wurde mit dem Simulationsmodell eines verketteten Produktionssystems (vgl. Abbildung 3 der Zusammenhang zwischen Ausfallzeit und Wartezeit bzw. Anzahl der unpünktlichen Güter untersucht. Von den beiden verschiedenen Produkten, die in der Anlage gefertigt werden, fährt Produkt 1 über Station 1, 3, 4 und 5 zur Senke, Produkt 2 fährt über Station 2, 3, und 4.

Ist eine Station belegt oder gestört, fährt das nächste Produkt eine Warteschleife. Die Leistungsverfügbarkeit wird über die Wartezeit an Quelle und Handarbeitsplatz (Station 4) und die unpünktlichen Güter an der Senke ermittelt.

In drei Versuchsreihen wurden zum einen der Redundanzfaktor Ri einiger Elemente variiert, in dem die Anteile von Produkt 1 und 2 verändert wurden und der Störungszeitpunkt TR wurde verändert. In Abbildung 4 und Abbildung 5 wird deutlich, dass die Struktur der Abhängigkeit den prognostizierten Graphen aus Abbildung 1 und Abbildung 2 entspricht. 


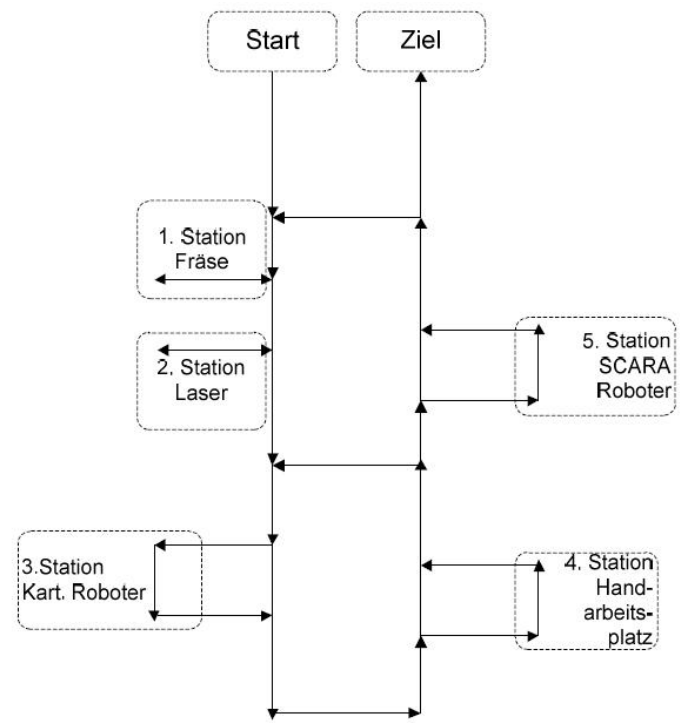

Abbildung 3: Simulationsmodell [Nag10, S. 20]

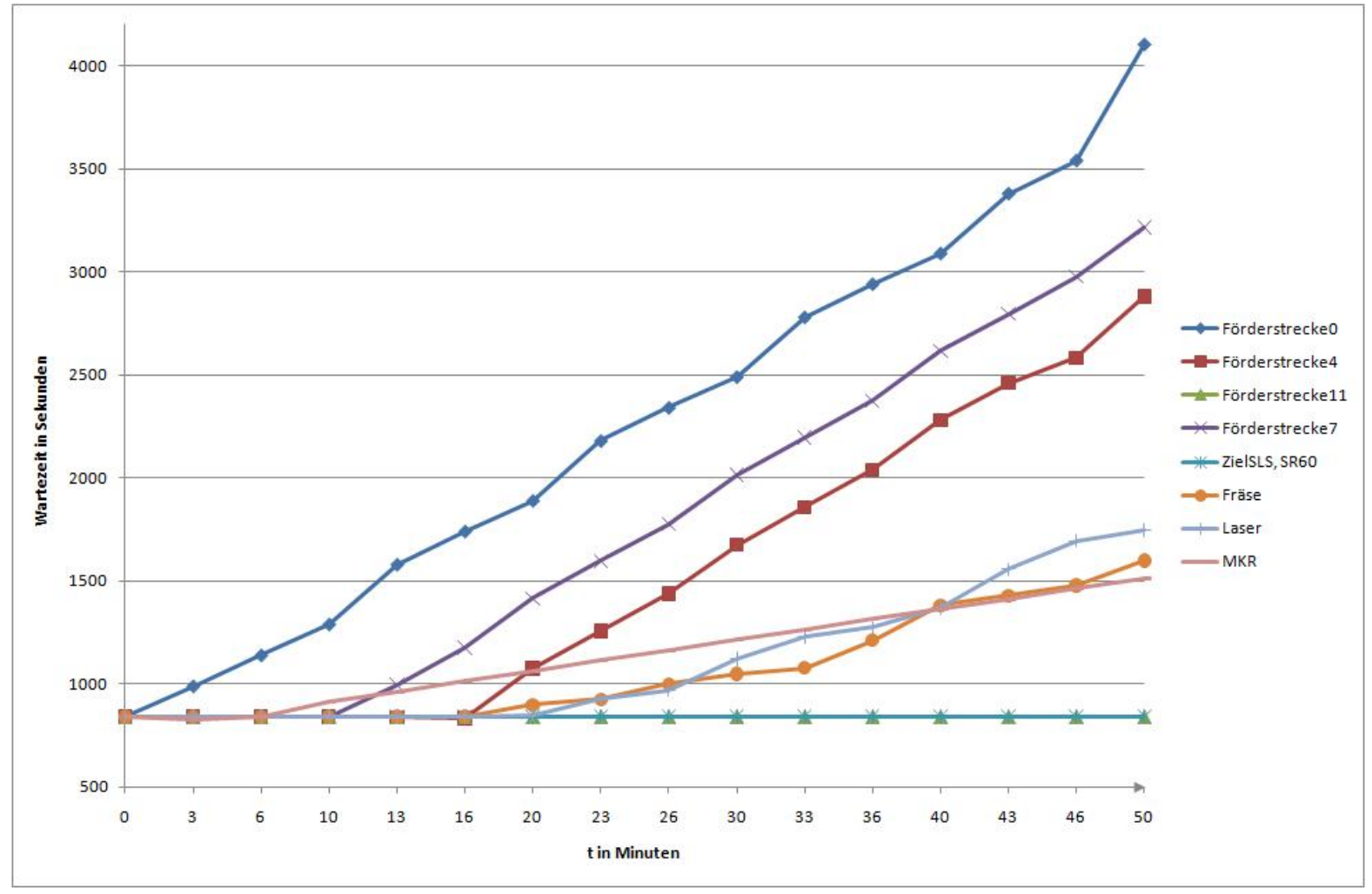

Abbildung 4: Simulationsergebnis Wartezeit [Nag10, S. 44] 


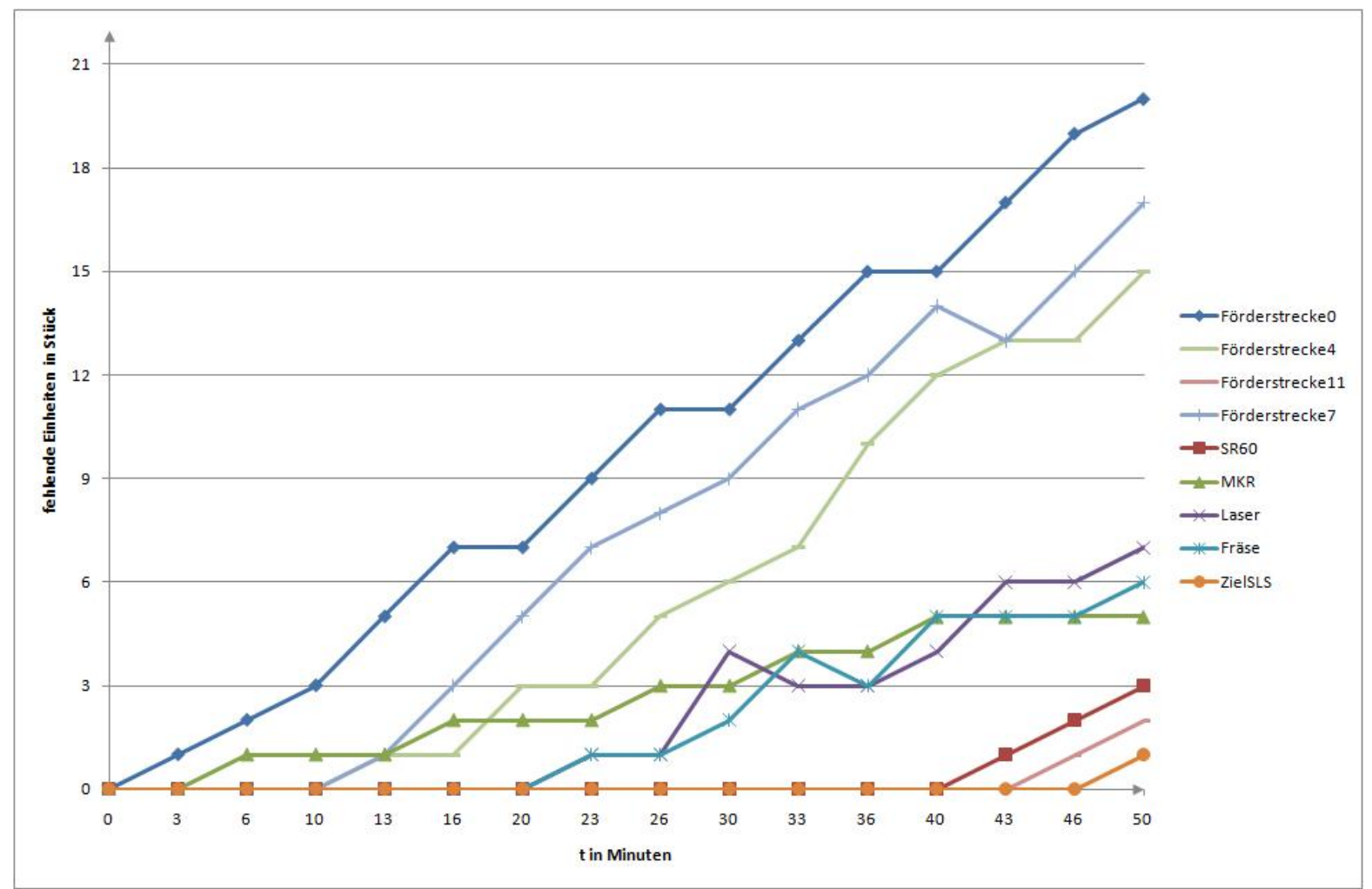

Abbildung 5: Simulationsergebnis unpünktlicher Güter [Nag10, S. 46]

Die Prognose der Steigung war sowohl bei Wartezeit als auch den unpünktlichen Gütern sehr gut (vgl. Tabelle 2); allerdings konnte der Einfluss des Reservekapazitätsfaktors auf die Steigung nicht validiert werden, da in der Steuerung des Systems keine Leistungssteigerung bei Störungen in parallelen Elementen vorgesehen war.

Tabelle 2: Prognosefehler Steigung

\begin{tabular}{c|ccc} 
& Datenanzahl & Mittl. Fehler & Standardabw. \\
\hline Wartezeit & 11 & $0,19 \mathrm{~min} / \mathrm{min}$ & $0,37 \mathrm{~min} / \mathrm{min}$ \\
Unp. Güter & 12 & 0,01 Stück $/ \mathrm{min}$ & 0,11 Stück $/ \mathrm{min}$
\end{tabular}

Für die Totzeit sind die Validierungsergebnisse schwankend. Bei der Wartezeitprognose waren $61 \%$ der Vorhersagen gut, bei den unpünktlichen Gütern 67\%. Die Abweichung erklärt sich aus folgenden Punkten: Die Totzeit ist von allen vier Parametern abhängig, von denen aber vor allem die Puffergrößen durch den Umlaufpuffer nicht exakt bestimmt werden konnten. Daneben haben Warteschleifen, die im Modell gefahren werden können, einen Einfluss auf das Ergebnis, sind aber in Praxis unüblich und daher nicht relevant für die Prognose. Da es sich aber um eine Methode zur Schätzung handelt, sind die Ergebnisse hinreichend genau.

\section{$4 \quad$ Anwendungsbeispiel: Lager}

Die vorgestellte Prognosemethode wird nun anhand einer Variantenauswahl für Vorzone eines Palettenlagers demonstriert. Berechnet werden jeweils die kritischen 
Ausfalldauern der Regalbediengeräte und der Techniklösung in der Vorzone. Weitere Kriterien für die Variantenauswahl, wie Kosten oder die Möglichkeit zur Erweiterung in laufenden Betrieb, werden nicht berücksichtigt.

\subsection{Basisdaten, Materialfluss und Anforderungen}

Es soll ein Palettenlager zur Versorgung der Montageabteilung geplant werden. Die Planung soll eine Erweiterungsstufe vorsehen. Das Geschäftsziel des Auftraggebers ist die auftragsgerechte Montage und Versand der Produkte (160-200 Produkte pro Tag, nach Erweiterung 240-300 Produkte pro Tag). Für die Hochregalanlage folgt daraus das primäre Ziel, die beiden Kommissionierarbeitsplätze auftragsgerecht zu versorgen und die Auftragspaletten termingerecht für die Montage bereit zu stellen. Ein nachrangiges Ziel ist die Aufnahme der am Wareneingang angelieferten Warenpaletten. Die Anlage arbeitet in zwei Schichten mit insgesamt 16 Stunden. Die Warenpaletten werden querschnittseingelagert. Die Auslagerungen folgen der FiFoStrategie. Nach längeren Havarien greift eine Wiederanlaufstrategie (vgl. Beitrag von Christian Wildner). Für die Instandhaltung sind während der Betriebszeit durchgängig zwei Mitarbeiter vor Ort.

Der Wareneingang wird unregelmäßig von ankommenden LKWs beliefert. Der Mitarbeiter am Wareneingang ist auch mit anderen Aufgaben betraut, er teilt sich die Arbeit selbstständig ein, allerdings müssen zum Schichtende, also nach 8 und nach 16 Stunden, alle bis dahin angelieferten Paletten auf die Fördertechnik aufgeben sein. Die Leistungsverfügbarkeit misst sich demnach an den nicht aufgegebenen Paletten gemäß Gleichung (2) bezogen auf die angelieferten Stück.

Für die Kommissioniermitarbeiter entsteht Wartezeit, wenn die geforderte Warenpalette nicht bereitsteht, oder die fertige Kundenpalette nicht weggefördert werden kann. Die Leistungsverfügbarkeit berechnet sich nach Gleichung (1). Die Auslagerungen erfolgen relativ gleichmäßig über den Tag. Der Mitarbeiter entnimmt die Paletten vom Auslagerstich und sie direkt zum jeweiligen Montagearbeitsplatz. Es entsteht Wartezeit, wenn nach der letzten Auslieferung keine Auslagerpalette bereitsteht. 


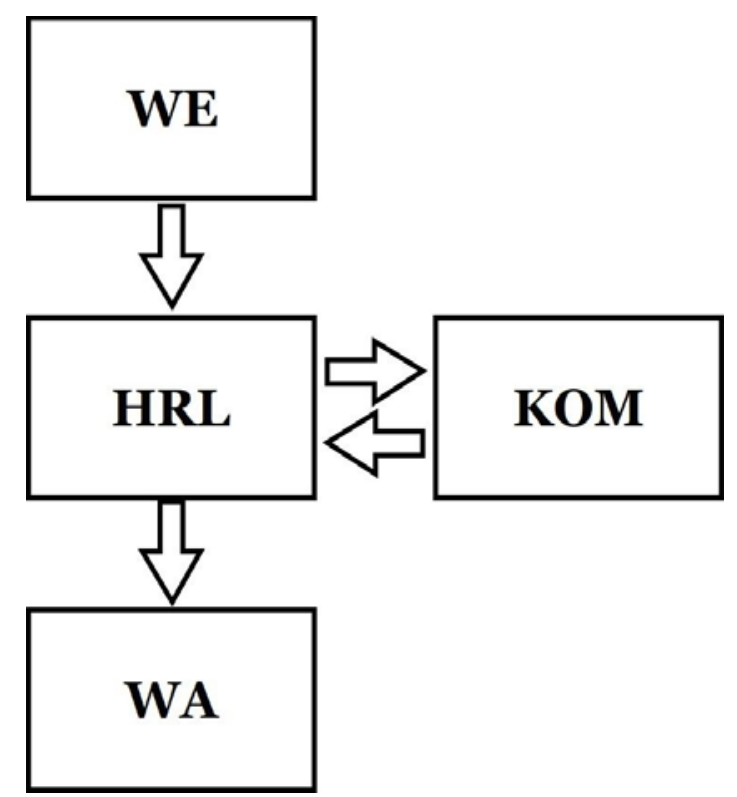

Abbildung 6: Schema

Der geplante Materialfluss wird schematisch in Abbildung 6 dargestellt und in Tabelle 3 quantifiziert.

Tabelle 3: Materialflussmatrix: Basisauslegung und (erweitert)

\begin{tabular}{|c|c|c|c|c|c|c|}
\hline & HRL & K1 & $\mathrm{K} 2$ & (K3) & $A L$ & Summe \\
\hline WE & $12(18)$ & & & & & 12(18) \\
\hline HRL & & 30 & 30 & (30) & $12(18)$ & 72(108) \\
\hline K1 & 30 & & & & & 30 \\
\hline K2 & 30 & & & & & 30 \\
\hline$\frac{\text { (K3) }}{\text { Summe }}$ & $\frac{(30)}{72(108)}$ & 30 & 30 & (30) & $12(18)$ & $\frac{(30)}{144(216)}$ \\
\hline
\end{tabular}

Die Leistungsverfügbarkeit (LV) des Lagers wird an den Arbeitsplätzen Wareneingang (WE), Kommissionierarbeitsplätze (K1-K3) und Auslagerpunkt (AL) gemessen, die Anforderungen sind in Tabelle 4 zusammengefasst.

Tabelle 4: Anforderungen an den Arbeitsplätzen

\begin{tabular}{c|cccc} 
Arbeitsplatz & Gleichung & Messzeit & Toleranz & LV \\
\hline WE & 2 & Zum Schichtende & Max. 3 Stück & $97 \%$ \\
K1, K2,(K3) & 1 & Pro Stunde & Max. 2 min & $96,7 \%$ \\
AL & 1 & Pro Stunde & Max. 1 min & $98,3 \%$
\end{tabular}

\subsection{Die Auslegung}

Zur Auswahl stehen für die Vorzone ein Verfahrwagen (VW), dessen Schiene bei Erweiterung verlängert wird, ein fahrerloses Transportsystem (FTS), für das bei Erweiterung zusätzliche Fahrzeuge verwendet werden und ein Stetigfördertechniksystem (StetigFT), das bei Erweiterung auf die neue Geometrie verlängert wird (vgl. Abbildung 7, 8 und 9). Zur Berechnung der kritischen Ausfallzeit werden in die Gleichungen (5) und (6) die Anforderungen aus Tabelle 4 eingesetzt und die Parameter für jeweils RBG und der Verteiltechnik aus Tabelle 5 verwendet. 
Tabelle 5: Berechnungsparameter

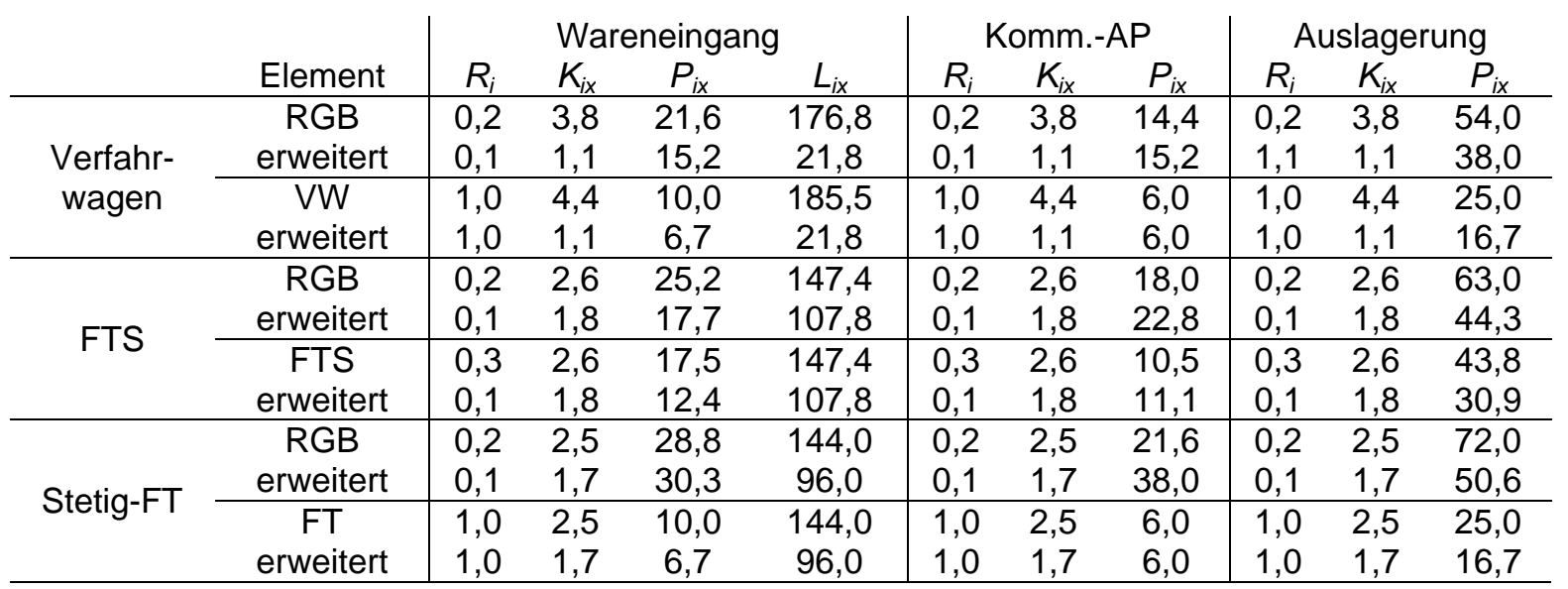

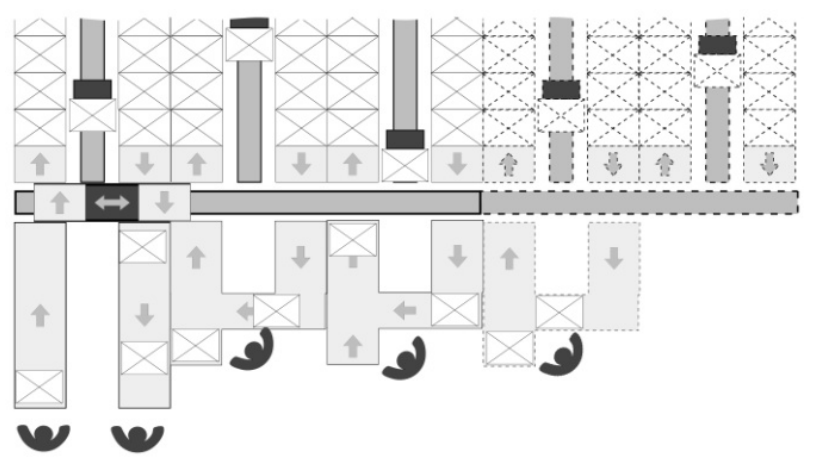

Abbildung 7: VW

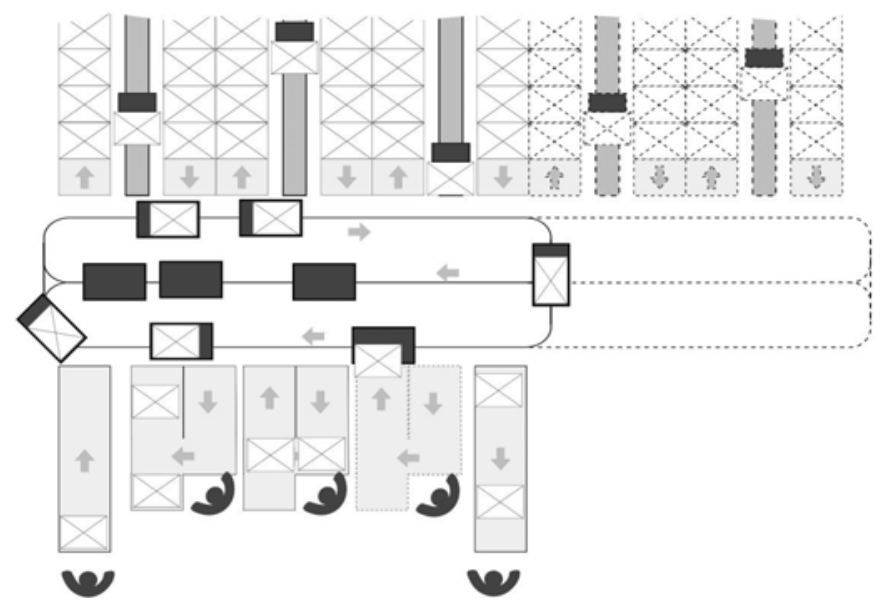

Abbildung 8: FTS

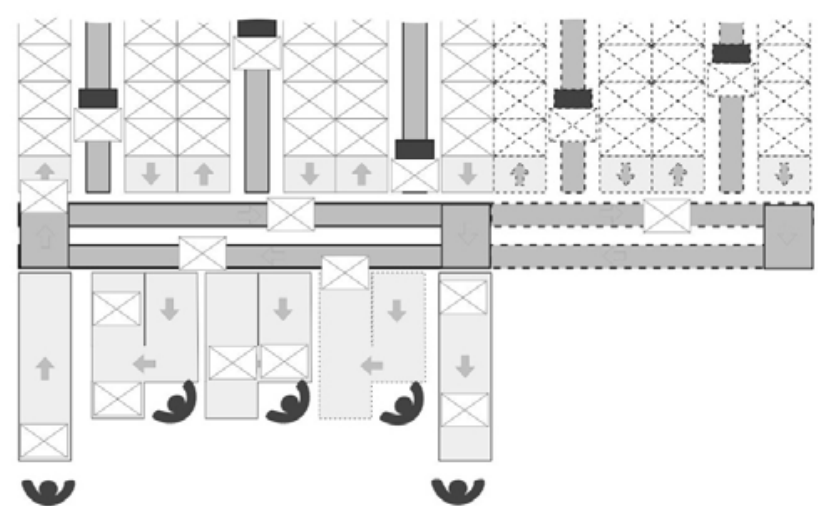

Abbildung 9: StetigFT 


\subsection{Prognoseergebnis}

Die kritischen Ausfallzeiten werden für die Regalbediengeräte in Abbildung 10 und die Verteiltechnik in Abbildung 11 dargestellt.

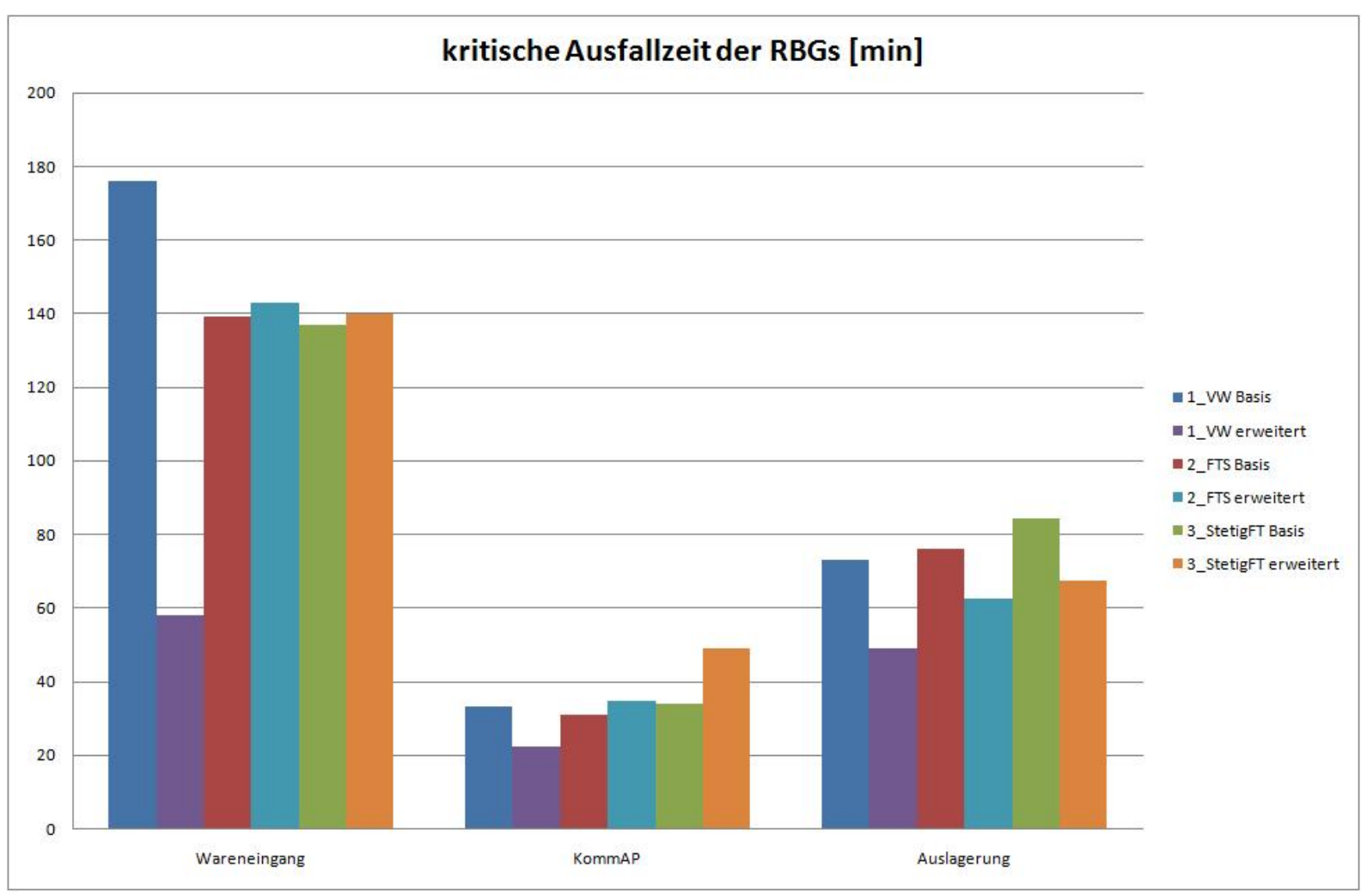

Abbildung 10: Kritische Ausfallzeiten der RBGs

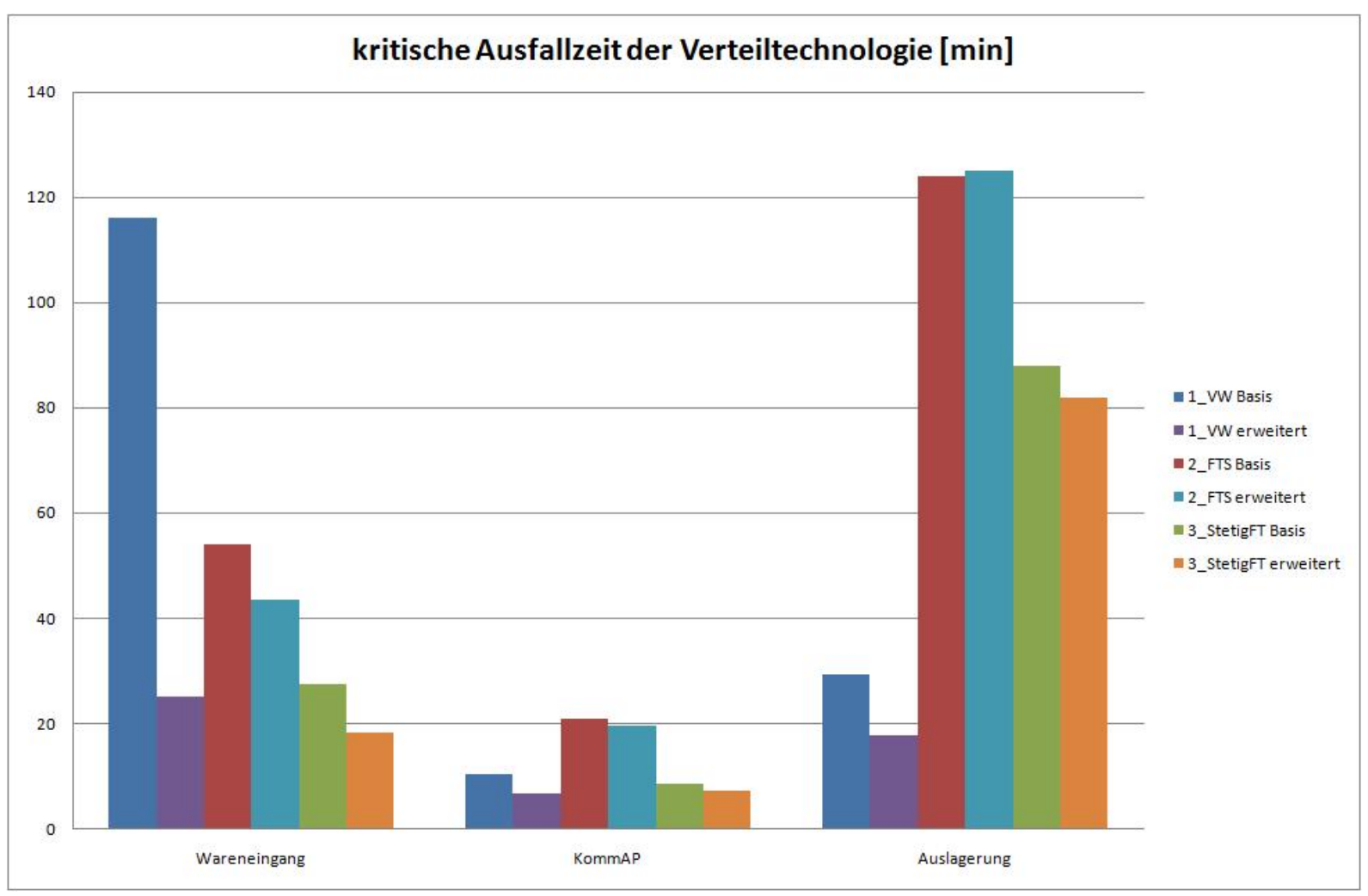

Abbildung 11: Kritische Ausfallzeiten der Verteiltechnik 
Es wird deutlich, dass die Kommissionierarbeitsplätze am anfälligsten für Störungsauswirkungen sind. Durch die Erweiterung verändern sich die zu Werte am stärksten beim Verfahrwagen hin zu deutlich kürzeren kritischen Ausfallzeiten, da dieser einen Großteil seiner Reservekapazität einbüßt. Bei Ausfällen der Regalbediengeräte hat die Stetigfördertechnik bedingt durch ihre zusätzliche Pufferfunktion die größte Möglichkeit, Störungen zu überbrücken. Bei einem Ausfall der Verteiltechnik ist das Lager mit dem fahrerlosen Transportsystem aufgrund der hohen Redundanz am Fehlertolerantesten.

\section{$5 \quad$ Fazit}

Die Prognosemethode ist in der Lage, die Leistungsverfügbarkeit einer geplanten Anlage mit hinreichender Genauigkeit abzuschätzen und Vergleiche zwischen verschiedenen Varianten zu ziehen. Außerdem kann der Bedarf von zusätzlichen Komponenten zur Verbesserung der zu erwartenden Leistungsverfügbarkeit deutlich gemacht werden. Es wird allerdings erneut darauf hingewiesen, dass es nicht zulässig ist, die Leistungsverfügbarkeit einer bestehenden Anlage aus den Störungsdaten mit den vorgestellten Abschätzungsgleichungen hochzurechnen. Hier gilt alleine das Messergebnis an den Schnittstellen.

\section{Literatur}

[Mai09] Maier, M. M.: Abnahmeprozeduren mit dem neuen Kennwert "Leistungsverfügbarkeit": angewandt auf die durchschnittliche, deutsche Kleinanlage. In: Scheid, W.-M. (Hrsg.): 5. Fachkolloquium der Wissenschaftlichen Gesellschaft für Technische Logistik (WGTL), Univ.Bibliothek, S. 219-230, 2009.

[Nag10] Nagel, M.: Simulation der Leistungsverfügbarkeit der Modellfabrik. Ilmenau: Technische Universität Ilmenau, Fakultät Maschinenbau, Bachelorarbeit, 2010.

[VDI92] VDI-Richtlinie 3649: Anwendung der Verfügbarkeitsrechnung für Förder und Lagersysteme. Januar 1992. - Inhaltlich überprüft und unverändert weiterhin gültig: August 2003. Berlin: Beuth Verlag, 1992.

[VDI04] VDI-Richtlinie 3581: Verfügbarkeit von Transport- und Lageranlagen sowie deren Teilsysteme und Elemente. Dezember 2004. Berichtigung der Richtlinie: Oktober 2006.

[VDI10] VDI Richtlinie 4486: Zuverlässigkeit in der Intralogistik: Leistungsverfügbarkeit. Berlin: Beuth Verlag, 2010. 